(C) The Authors 2017. This is an Open Access article, distributed under the terms of the Creative

Commons Attribution-NonCommercial-ShareAlike licence (http://creativecommons.org/licenses/by-nc-sa/4.0/),

which permits non-commercial re-use, distribution, and reproduction in any medium, provided the same

Creative Commons licence is included and the original work is properly cited. The written permission of

Cambridge University Press must be obtained for commercial re-use.

\title{
Heat-stabilised rice bran consumption by colorectal cancer survivors modulates stool metabolite profiles and metabolic networks: a randomised controlled trial
}

\author{
Dustin G. Brown ${ }^{1}$, Erica C. Borresen ${ }^{1}$, Regina J. Brown ${ }^{2,3}$ and Elizabeth P. Ryan ${ }^{1,3 *}$ \\ ${ }^{1}$ Department of Environmental and Radiological Health Sciences, Colorado State University, Fort Collins, CO 80521, USA \\ ${ }^{2}$ Division of Medical Oncology, University of Colorado School of Medicine, Aurora, CO 80045, USA \\ ${ }^{3}$ University of Colorado Cancer Center, Aurora, CO 80045, USA
}

(Submitted 28 October 2016 - Final revision received 28 March 2017 - Accepted 11 April 2017)

\begin{abstract}
Rice bran (RB) consumption has been shown to reduce colorectal cancer (CRC) growth in mice and modify the human stool microbiome. Changes in host and microbial metabolism induced by RB consumption was hypothesised to modulate the stool metabolite profile in favour of promoting gut health and inhibiting CRC growth. The objective was to integrate gut microbial metabolite profiles and identify metabolic pathway networks for CRC chemoprevention using non-targeted metabolomics. In all, nineteen CRC survivors participated in a parallel randomised controlled dietary intervention trial that included daily consumption of study-provided foods with heat-stabilised RB ( $30 \mathrm{~g} / \mathrm{d}$ ) or no additional ingredient (control). Stool samples were collected at baseline and 4 weeks and analysed using GC-MS and ultra-performance liquid chromatography-MS. Stool metabolomics revealed 93 significantly different metabolites in individuals consuming RB. A 264-fold increase in $\beta$-hydroxyisovaleroylcarnitine and 18-fold increase in $\beta$-hydroxyisovalerate exemplified changes in leucine, isoleucine and valine metabolism in the RB group. A total of thirty-nine stool metabolites were significantly different between RB and control groups, including increased hesperidin (28-fold) and narirutin (14-fold). Metabolic pathways impacted in the RB group over time included advanced glycation end products, steroids and bile acids. Fatty acid, leucine/valine and vitamin $\mathrm{B}_{6}$ metabolic pathways were increased in RB compared with control. There were 453 metabolites identified in the RB food metabolome, thirty-nine of which were identified in stool from RB consumers. RB consumption favourably modulated the stool metabolome of CRC survivors and these findings suggest the need for continued dietary CRC chemoprevention efforts.
\end{abstract}

Key words: Colorectal cancer: Rice bran: Metabolomics: Phytochemicals: Metabolic pathways

Convincing evidence supports that foods rich in phytochemicals and dietary fibre decrease risk of colorectal cancer $(\mathrm{CRC})^{(1,2,3)}$. With CRC being the third most common cancer in the world with approximately 1.4 million new cases ${ }^{(4)}$, a focus on lifestyle behaviours, especially diet, may be beneficial for cancer prevention. Dietary fibre is comprised of NSP that are found in fruits, vegetables, legumes and whole grains ${ }^{(1)}$. Phytochemicals are diverse classes of compounds including, but not limited to antioxidants, polysaccharides and saponins ${ }^{(2)}$. Whole grains are a rich source of dietary fibre and phytochemicals that have been studied for cancer chemopreventive properties ${ }^{(5,6)}$. Whole grain brown rice and rice bran (RB) contain multiple unique plant bioactive components (i.e. $\beta$-sitosterol, $\gamma$-oryzanol, vitamin $\mathrm{E}$ isoforms, prebiotics and dietary fibres) that have been shown to inhibit CRC growth ${ }^{(7-10)}$. For example, consuming $30 \%$ fibre from bran had a chemopreventive effect of reduced adenoma burden in mice, equating to approximately $1.2 \mathrm{~g} / \mathrm{d}$ per kg RB fibre for human consumption ${ }^{(11)}$.

Dietary RB elicits chemopreventive actions via multiple mechanisms, such as inhibition of cyclo-oxygenase 2, induced nitric oxide synthase expression, $\mathrm{NF}-\kappa \mathrm{B}$ signalling, chromatin condensation and caspase 3 activation, among others ${ }^{(12-14)}$ RB metabolism by gut microflora may decrease the intestinal $\mathrm{pH}$ and increase intestinal SCFA production ${ }^{(15-17)}$. During colonic fermentation, there are distinct interactions between host and microbial metabolism, resulting in production of secondary metabolites that originated from RB intake ${ }^{(18)}$. Modulation of substrates available to gut microbiota from the diet can influence the type and amount of microbial metabolites produced, and was correlated with various health outcomes, including cancer $^{(19)}$. Despite the established dietary chemopreventive actions of $\mathrm{RB}$, a knowledge gap remains of the profile of $\mathrm{RB}$

Abbreviations: AGE, advanced glycation end product; CRC, colorectal cancer; RB, Rice bran; UPLC, ultra-performance liquid chromatography.

* Corresponding author: E. P. Ryan, email E.P.Ryan@colostate.edu 
small molecules that reach the colon and that work in an additive or synergistic manner to promote healthy metabolism and gastrointestinal functions ${ }^{(20)}$.

Clinical trials established the feasibility of increased consumption of heat-stabilised RB in people and showed that RB intake can modulate the human stool microbiome ${ }^{(15,21,22)}$. These studies helped determine what phytochemicals from RB interact for enhanced microbial metabolism in the colon and excretion in stool. The objective of this study was to integrate gut microbial metabolite profiles and identify metabolic pathway networks for CRC chemoprevention using non-targeted metabolomics. We hypothesised that stool metabolites resulting from both host and microbial metabolism following dietary RB intake represent viable, chemopreventive agents for inhibition of colonic inflammation and CRC recurrence. Results from this study have important dietary implications for prevention of primary and secondary CRC recurrence in adults.

\section{Methods \\ Study population and stool sample collection}

CRC survivors were recruited and enrolled in a parallel randomised controlled dietary intervention trial (NCT01929122) as previously described $^{(21)}$. In brief, CRC survivors were deemed eligible if they were a healthy adult with a history of CRC, a minimum of 4 months post-cancer treatment, able to follow a dietary intervention for $28 \mathrm{~d}$, had no history of food allergies or major dietary restrictions, and had a BMI between 25 and $35 \mathrm{~kg} / \mathrm{m}^{2}$. Participants consumed study-provided foods which included one meal and one snack each day. All study meals and snacks were calorically matched across study groups and previously described as including either $30 \mathrm{~g} / \mathrm{d}$ of heatstabilised $\mathrm{RB}$ or no additional ingredient (control) ${ }^{(21)}$. The addition of $30 \mathrm{~g} \mathrm{RB}$ to the meal and snack provided $397 \mathrm{~kJ}$ (95 kcal) and $6 \mathrm{~g}$ of total dietary fibre daily. The consumption of one study meal and snack each day ranged between approximately 2636 and $2929 \mathrm{~kJ} / \mathrm{d}$ (630 and $700 \mathrm{kcal} / \mathrm{d}$ ), about onethird of total energy content consumed daily. The remaining two-thirds of daily energy content, approximately $5858 \mathrm{~kJ}$ (1400 kcal), was not controlled and was analysed accordingly with the participant's typical dietary intake. To track all foods consumed, participants recorded dietary intakes using 3-d food logs, which included records of the study-provided foods. These food logs were analysed using Nutrition Pro ${ }^{\mathrm{TM}}$ (Axxya Systems) and nutrition results have been previously reported $^{(21)}$.

This study was conducted according to the guidelines laid down in the Declaration of Helsinki and all procedures involving human participants were approved by Poudre Valley Hospital/University of Colorado Health - North Institutional Review Board (Protocol no. 10-1038) and Colorado State University Institutional Review Board (Protocol no. 09-1520H). Written informed consent was obtained from all participants. Stratified randomisation was completed based on sex, BMI and energy intake and participants were randomised into either a control diet group or RB diet group. Study-provided meals and snacks were coded to ensure participants remained blinded to their diet group. The study took place at the University of Colorado Health - North Cancer Center in Fort Collins, CO, between August 2010 and December 2014. Participants selfcollected stool samples in specimen containers at two time points: baseline and week 4 . Stool samples were lyophilised, aliquoted and stored at $-80^{\circ} \mathrm{C}$ before metabolite extraction. The online Supplementary Fig. S1 illustrates the study flow.

\section{Heat-stabilised rice bran for dietary intervention and metabolomics}

The US Department of Agriculture-Rice Research Center provided the heat-stabilised RB used in this study. Rice paddy was milled using standard milling processes to isolate bran. Following milling, RB was heat-stabilised at $110^{\circ} \mathrm{C}$ for $3 \mathrm{~min}$ and stored at $-20^{\circ} \mathrm{C}$. For this study, Neptune and Dixiebell varieties were chosen due to agronomic similarities and incorporated into study meals and snacks and their nutritional composition has been previously described ${ }^{(21,22)}$. For RB food metabolome determination, $100 \mathrm{mg}$ of $\mathrm{RB}$ was subjected to non-targeted metabolite profiling at Metabolon. RB was extracted with $80 \%$ methanol as previously described, and analysed by GC-MS and ultra-performance liquid chromatography-MS (UPLC-MS/MS) in the positive and negative ionisation mode platforms ${ }^{(23)}$.

\section{Stool extractions for metabolomics}

Stool samples were extracted for metabolite profile evaluation by Metabolon Inc. as previously described ${ }^{(24)}$. Stool samples were extracted using $80 \%$ methanol. The final extract was divided into four aliquots for: UPLC-MS/MS Positive Ion Mode, UPLC-MS/MS Negative Ion Mode, GC-MS and backup.

\section{GC-MS}

Samples were derivatised under $\mathrm{N}_{2}$ using bistrimethylsilyltrifluoroacetamide and separated on a $5 \%$ diphenyl $/ 95 \%$ dimethyl polysiloxane fused silica column $(20 \mathrm{~m} \times 0 \cdot 18 \mathrm{~mm}$ ID; $0 \cdot 18 \mu \mathrm{m}$ film thickness) with $\mathrm{He}$ as carrier gas and a temperature ramp from 60 to $340^{\circ} \mathrm{C}$ in a $17.5 \mathrm{~min}$ period. Internal standards amylbenzene, 1-phenylhexane, 1- phenyloctane, 1-phenyldecane, 1-phenyldodecane, hexadecylbenzene, octadecylbenzene, tetradecylbenzene and 2,6-di-tert-butyl-4-methylphenol were added to each sample ( $250 \mathrm{ng}$ of each standard per sample). Samples were analysed on a Thermo-Finnigan Trace DSQ fastscanning singlequadrupole mass spectrometer using electro impact ionisation and operated at unit mass resolving power. The scan range was from 50 to $750 \mathrm{~m} / \mathrm{z}$.

\section{Ultra-performance liquid chromatography-MS}

The UPLC-MS/MS portion of the platform was based on a Waters ACQUITY UPLC and a Thermo-Finnigan LTQ MS operated at nominal mass resolution, which consisted of an electrospray ionisation source and linear ion-trap mass analyser. The dried sample extract was reconstituted in acidic or basic UPLC-compatible solvents, each of which contained eleven to thirteen injection standards at fixed concentrations ${ }^{(25)}$. One aliquot was analysed using acidic ten positive ion-optimised 
conditions and the other using basic, negative ion-optimised conditions in two independent injections using separate dedicated columns (UPLC BEH C18-2.1 $\times 100 \mathrm{~mm}, 1 \cdot 7 \mu \mathrm{m}$; Waters). Extracts reconstituted in acidic conditions were gradient eluted using water and methanol containing $0 \cdot 1 \%$ formic acid, whereas the basic extracts, which also used water/methanol, contained $6.5 \mathrm{~mm}$ ammonium bicarbonate. The MS analysis alternated between MS and data-dependent MS/MS scans using dynamic exclusion and the scan range was from 80 to $1000 \mathrm{~m} / \mathrm{z}$. Raw data were extracted, peak-identified and quality control processed as previously described ${ }^{(24)}$.

\section{Metabolic pathway networks and analysis}

To visualise relevant networks of metabolic pathways from stool metabolites, the relative abundances were uploaded into a pathway analysis software and metabolite classification system called Metabolync (portal.metabolon.com). Pathway impact scores were calculated as previously described, and takes into account the number of significantly different compounds in a pathway with all metabolites identified ${ }^{(24)}$. A score $\geq 2$ indicates significant alterations of a metabolic pathway when compared with other pathways. Metabolites were visualised via Cytoscape version 2.8.3. Each metabolite was represented by a closed, coloured node extending from a sub-metabolic pathway node (via pathway enrichment score), which connect to a supermetabolic pathway hexagonal node. A node coloured red represents metabolites with significantly higher expression at 4 weeks compared with baseline $(P \leq 0 \cdot 05)$. A node coloured dark green represents metabolites with significantly lower expression at 4 weeks compared with baseline. Nodes coloured pink or light green represent metabolites trending towards significance with higher expression at 4 weeks and lower expression at 4 weeks, respectively $(0 \cdot 05<P \leq 0 \cdot 1)$. Node size is proportional to fold change magnitude over the 4 -week time period for RB diet group.

\section{Statistical analyses}

$\mathrm{RB}$ and stool metabolite profiles from CRC survivors were quantified in terms of relative abundance and median scaled to 1 as previously described ${ }^{(24)}$. Metabolomics as an endpoint can be difficult to calculate power due to the complexity of metabolomics studies, including the constant expansion of metabolite libraries and the large number of metabolites identified. There are currently no accepted methods for sample size calculations ${ }^{(26,27)}$. However, a previous trial in seven healthy adults with no history of CRC, with four participants consuming RB found detectable stool metabolome changes ${ }^{(15)}$. Thus, a sample group of approximately ten is a reasonable sample size to detect significant changes.

Statistics were performed in ArrayStudio (Omicsoft), GraphPad Prism 6.06 and SAS version 9.4. $P$ values of $\leq 0.05$ were statistically significant. A matched-pairs two-way ANOVA with post-test contrasts was completed using the scaled relative abundance of each metabolite to evaluate the impact of dietary RB supplementation over 4 weeks' time and with regards to changes in the stool metabolome. An estimate of false discovery rate ( $q$ value) was calculated to determine false discoveries common to multiple-comparison-based metabolomics studies. Metabolites with $q$ values $\geq 0 \cdot 1$ were excluded from further $\operatorname{analysis}^{(24)}$.

\section{Results}

A total of nineteen CRC survivors were randomised to a diet intervention group that was either $30 \mathrm{~g} / \mathrm{d}$ of RB or none (control). In all, twenty-five participants were initially randomised to control ( $n$ 10) or RB ( $n$ 15). Six participants withdrew from the trial due to non-compliance ( $n$ 5) and family emergency $(n 1)$. No adverse events were reported. Participant characteristics at baseline are presented in the online Supplementary Table S1.

\section{Rice bran modulation of stool metabolite profiles and metabolic pathway networks}

The principal component analysis of baseline and 4-week time points revealed inter-individual stool metabolome differences and minor clustering of individuals after 4 weeks of RB intake (online Supplementary Fig. S2). Table 1 lists ninety-three significant stool metabolites from CRC survivors consuming RB at 4 weeks compared with baseline. Stool metabolites are presented as the mean fold change or mean fold difference with respect to the scaled relative abundance of each metabolite for all participants. Of the ninety-three stool metabolites changed with RB consumption, thirty-three metabolites increased and sixty metabolites decreased after 4 weeks (Fig. 1(a) and (b)). $\beta$-Hydroxyisovaleroylcarnitine and hydroxybutyrlcarnitine showed a 265 -fold and 66-fold increase over time, respectively. In addition, hesperidin (37-fold), apigenin (15-fold), narirutin (15-fold), hesperitin (12-fold), enterodiol (11-fold), diosmetin (5-fold), enterolactone (4-fold), eriocitrin (3-fold) and homostachydrine (1.14-fold) were reflective of increased phytochemical consumption and differentially expressed in the stool metabolome.

Metabolites were classified into metabolic pathway networks and analysed by diet group and time. At 4 weeks, a total of eight metabolic pathways were affected by RB consumption (Fig. 2(a)). Pathways included advanced glycation end product (AGE) (pathway impact score of 8.69), steroid metabolism (5.04), primary bile acid metabolism (2.9), leucine, isoleucine and valine metabolism (2.79), methionine, cysteine, $S$-adenosyl methionine and taurine metabolism (2.29), inositol metabolism (2.17), vitamin $\mathrm{B}_{6}$ metabolism (2.17) and benzoate metabolism (2.0) (Fig. 2(a)).

\section{Visual metabolite pathway classification and nodal networks for rice bran consumers}

Fig. 3 and 4 display Cytoscape pathway classification nodal network views of the stool metabolic pathways modulated by RB consumption at 4 weeks compared with baseline levels. This visual representation includes metabolites from lipid, cofactor and vitamins metabolic pathways in RB consumers (Fig. 3(a)-(d)). Carbohydrates and amino acid pathways are shown in Fig. 4(a)-(d). Network views confirmed the ninetythree metabolites significantly modulated by RB intake and the suite of metabolites trending towards significance. Fig. 3(a) 
Table 1. Stool metabolites distinguishing rice bran consumption at 4 weeks compared to baseline

\begin{tabular}{|c|c|c|c|c|}
\hline Chemical classes & Metabolic pathway & Metabolite & $P$ & $\begin{array}{c}\text { Fold Change } \\
\text { (4 weeks/baseline) }\end{array}$ \\
\hline \multirow[t]{29}{*}{ Amino acid } & Glutathione metabolism & S-methylglutathione & 0.0048 & $0.4 \downarrow$ \\
\hline & \multirow[t]{2}{*}{ Histidine metabolism } & Formiminoglutamate & 0.0127 & $0.21 \downarrow$ \\
\hline & & $N$-acetylhistamine & 0.0367 & $0.52 \downarrow$ \\
\hline & \multirow{9}{*}{$\begin{array}{l}\text { Leucine, isoleucine and valine } \\
\text { metabolism }\end{array}$} & $\beta$-Hydroxyisovaleroylcarnitine & 0.0417 & $264.52 \uparrow$ \\
\hline & & $\beta$-Hydroxyisovalerate & 0.033 & $17.9 \uparrow$ \\
\hline & & 4-methyl-2-oxopentanoate & 0.0066 & $0.38 \downarrow$ \\
\hline & & 3-methyl-2-oxobutyrate & 0.0129 & $0.36 \downarrow$ \\
\hline & & 3-methyl-2-oxovalerate & 0.0122 & $0.36 \downarrow$ \\
\hline & & Allo-isoleucine & 0.0005 & $0.31 \downarrow$ \\
\hline & & Ethylmalonate & 0.0475 & $0.7 \downarrow$ \\
\hline & & Isovalerylglycine & 0.0279 & $0 \cdot 7 \downarrow$ \\
\hline & & $N$-acetylleucine & 0.0324 & $0.63 \downarrow$ \\
\hline & \multirow{3}{*}{ Lysine metabolism } & Glutarylcarnitine (C5) & 0.0331 & $48 \cdot 32 \uparrow$ \\
\hline & & Pipecolate & 0.0232 & $0.49 \downarrow$ \\
\hline & & N6-carboxyethyllysine & 0.0011 & $0.39 \downarrow$ \\
\hline & \multirow{5}{*}{$\begin{array}{l}\text { Methionine, cysteine, S-adenosyl } \\
\text { methionine and } \\
\text { taurine metabolism }\end{array}$} & Cystathionine & 0.0277 & $1.9 \uparrow$ \\
\hline & & $N$-formylmethionine & 0.0443 & $1 \cdot 1 \uparrow$ \\
\hline & & $N$-acetylmethionine & 0.0194 & $0.88 \downarrow$ \\
\hline & & $\mathrm{N}$-acetylmethionine sulfoxide & 0.0043 & $0.47 \downarrow$ \\
\hline & & 4-methylthio-2-oxobutanoate & 0.013 & $0 \cdot 22 \downarrow$ \\
\hline & \multirow{5}{*}{$\begin{array}{l}\text { Phenylalanine and tyrosine } \\
\text { metabolism }\end{array}$} & Phenol sulfate & 0.0303 & $4 \cdot 68 \uparrow$ \\
\hline & & $p$-cresol sulfate & 0.0376 & $0.69 \downarrow$ \\
\hline & & $N$-formylphenylalanine & 0.0163 & $0.65 \downarrow$ \\
\hline & & 4-hydroxyphenylpyruvate & 0.0021 & $0.4 \downarrow$ \\
\hline & & Phenylpyruvate & 0.0081 & $0.32 \downarrow$ \\
\hline & Tryptophan metabolism & Xanthurenate & $1 \cdot 20 \mathrm{E}-05$ & $4 \cdot 27 \uparrow$ \\
\hline & \multirow{3}{*}{$\begin{array}{l}\text { Urea cycle; arginine and proline } \\
\text { metabolism }\end{array}$} & Citrulline & 0.0173 & $0.67 \downarrow$ \\
\hline & & $N$-methylproline & 0.0386 & $0.43 \downarrow$ \\
\hline & & Homocitrulline & 0.0252 & $0.39 \downarrow$ \\
\hline \multirow{2}{*}{ Carbohydrate } & Advanced glycation end product & N6-carboxymethyllysine & 0.0059 & $0.62 \downarrow$ \\
\hline & Pentose metabolism & Xylitol & 0.0072 & $2 \cdot 11 \uparrow$ \\
\hline \multirow[t]{2}{*}{ Cofactors and vitamins } & Tocopherol metabolism & $\gamma$-Carboxyethyl-hydroxychroman & 0.0243 & $1.78 \uparrow$ \\
\hline & Vitamin $\mathrm{B}_{6}$ metabolism & Pyridoxine (vitamin $\mathrm{B}_{6}$ ) & 0.0102 & $2.96 \uparrow$ \\
\hline Energy & TCA cycle & Aconitate (cis or trans) & 0.0117 & $11 \cdot 12 \uparrow$ \\
\hline \multirow[t]{34}{*}{ Lipid } & \multirow[t]{2}{*}{ Fatty acid metabolism(acyl carnitine) } & Hydroxybutyrylcarnitine* & 0.0106 & $65 \cdot 96 \uparrow$ \\
\hline & & Hexanoylcarnitine & 0.0306 & $32 \cdot 74 \uparrow$ \\
\hline & \multirow[t]{3}{*}{ Fatty acid, dicarboxylate } & Hexadecanedioate & 0.0042 & $2 \cdot 07 \uparrow$ \\
\hline & & Sebacate (decanedioate) & 0.0485 & $0.62 \downarrow$ \\
\hline & & Azelate (nonanedioate) & 0.035 & $0.55 \downarrow$ \\
\hline & Fatty acid, monohydroxy & 2-hydroxyoctanoate & 0.0391 & $0 \cdot 24 \downarrow$ \\
\hline & & $a$-Hydroxycaproate & 0.0396 & $0 \cdot 17 \downarrow$ \\
\hline & Inositol metabolism & Chiro-inositol & 0.0251 & $26 \cdot 15 \uparrow$ \\
\hline & Medium-chain fatty acid & 5-dodecenoate (12:1n-7) & 0.035 & $2.58 \uparrow$ \\
\hline & Phospholipid metabolism & Ethanolamine & 0.0148 & $1.74 \uparrow$ \\
\hline & & Pelargonate $(9: 0)$ & 0.0456 & $0.45 \downarrow$ \\
\hline & PUFA ( $n-3$ and $n-6)$ & Dihomo-linolenate $(20: 3 n-3$ or $n-6)$ & 0.0367 & $0.94 \downarrow$ \\
\hline & Primary bile acid metabolism & Glycochenodeoxycholate & 0.0421 & $0.27 \downarrow$ \\
\hline & & Taurocholate & 0.019 & $0.26 \downarrow$ \\
\hline & Secondary bile acid metabolism & Glycodeoxycholate & 0.0344 & $0.22 \downarrow$ \\
\hline & Steroid & 4-androsten-3a,17a-diol monosulfate (2) & 0.0165 & $9 \cdot 49 \uparrow$ \\
\hline & & 4-androsten-3 $\beta, 17 \beta$-diol monosulfate (2) & 0.033 & $3.07 \uparrow$ \\
\hline & & $5 a$-androstan- $3 \beta, 17 a$-diol disulfate & 0.0345 & $0.62 \downarrow$ \\
\hline & & 11-ketoetiocholanolone sulfate & 0.0004 & $0.54 \downarrow$ \\
\hline & & Pregnanolone/allopregnanolone sulfate & 0.0476 & $0.45 \downarrow$ \\
\hline & & Epiandrosterone sulfate & 0.0289 & $0.41 \downarrow$ \\
\hline & & 4-androsten- $3 \beta, 17 \beta$-diol disulfate (1) & 0.0012 & $0.35 \downarrow$ \\
\hline & & 4-androsten- $3 \beta, 17 \beta$-diol disulfate (2) & 0.0042 & $0.35 \downarrow$ \\
\hline & & $5 \alpha$-androstan- $3 \beta, 17 \beta$-diol monosulfate (2) & 0.006 & $0.26 \downarrow$ \\
\hline & & $5 a$-pregnan- $3 \beta, 20 a$-diol disulfate & 0.0009 & $0 \cdot 25 \downarrow$ \\
\hline & & $5 \alpha$-androstan- $3 \beta, 17 \beta$-diol disulfate & 0.0049 & $0 \cdot 23 \downarrow$ \\
\hline & & Dehydroisoandrosterone sulfate (DHEA-S) & 0.0013 & $0.22 \downarrow$ \\
\hline & & Pregnenolone sulfate & 0.0109 & $0 \cdot 21 \downarrow$ \\
\hline & & Pregnen-diol disulfate* & 0.0235 & $0 \cdot 19 \downarrow$ \\
\hline & & $5 a$-pregnan-3( $a$ or $\beta), 20 \beta$-diol disulfate & 0.0112 & $0 \cdot 14 \downarrow$ \\
\hline & & 16a-hydroxy DHEA 3-sulfate & 0.0086 & $0 \cdot 14 \downarrow$ \\
\hline & & 4 -androsten- $3 \beta, 17 \beta$-diol monosulfate (1) & 0.0142 & $0 \cdot 13 \downarrow$ \\
\hline & & Pregn steroid monosulfate* & 0.0109 & $0 \cdot 12 \downarrow$ \\
\hline & Sterol & 4-cholesten-3-one & 0.0434 & $0.58 \downarrow$ \\
\hline
\end{tabular}


Table 1. Continued

\begin{tabular}{|c|c|c|c|c|}
\hline Chemical classes & Metabolic pathway & Metabolite & $P$ & $\begin{array}{c}\text { Fold Change } \\
\text { (4 weeks/baseline) }\end{array}$ \\
\hline $\begin{array}{l}\text { Peptide } \\
\text { Other }\end{array}$ & Phytochemical & $\begin{array}{l}\gamma \text {-Glutamylphenylalanine } \\
\text { 2-hydroxyhippurate (salicylurate) } \\
\text { 3-(2-hydroxyphenyl)propionate } \\
p \text {-aminobenzoate (PABA) } \\
\text { 2-oxo-1-pyrrolidinepropionate } \\
\text { 3-aminobutyrate } \\
\text { Brilliant blue FCF (blue 1) } \\
\text { Diosmin } \\
\text { Salicylate } \\
\text { Metoprolol acid metabolite* } \\
\text { Ranitidine } \\
\text { Hesperidin } \\
\text { Apigenin } \\
\text { Narirutin } \\
\text { Hesperetin } \\
\text { Enterodiol } \\
\text { Diosmetin } \\
\text { Enterolactone } \\
\text { Eriocitrin } \\
\text { Homostachydrine* } \\
\text { Abscisate } \\
\text { Capsaicin } \\
\text { Harmane } \\
\text { Methyl indole-3-acetate } \\
\text { Solanidine }\end{array}$ & $\begin{array}{l}0.0428 \\
3.83 E-05 \\
0.0386 \\
0.0485 \\
0.0435 \\
0.045 \\
0.0492 \\
0.0018 \\
0.0417 \\
0.0103 \\
0.01 \\
0.0017 \\
0.0002 \\
0.0089 \\
0.0329 \\
0.0013 \\
0.0347 \\
0.0015 \\
0.0114 \\
0.0356 \\
0.0475 \\
0.0135 \\
0.0407 \\
0.0082 \\
4.98 E-05\end{array}$ & 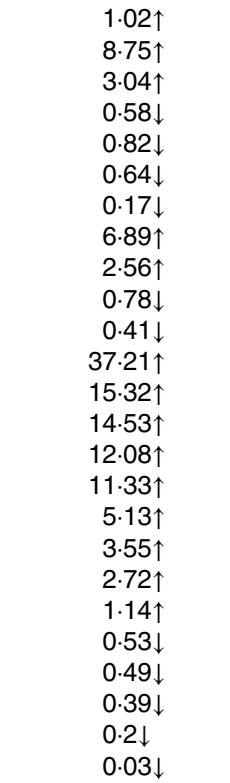 \\
\hline
\end{tabular}

$\uparrow$, Metabolites with significantly $(P \leq 0.05)$ higher expression in rice bran consumers at 4 weeks when compared with rice bran consumers at baseline (metabolite ratio of $\geq 1.00)$; $\downarrow$, metabolites with significantly $(P \leq 0.05)$ lower expression in rice bran consumers at 4 weeks when compared with rice bran consumers at baseline (metabolite ratio of $<1.00)$. * Compounds that have not been officially confirmed based on a standard, but we are confident in its identity.

shows the steroid metabolic pathway which was significantly affected after RB consumption with sixteen metabolites significantly decreased. An increase in 4-andorsten- $3 \alpha, 17 \alpha$-diol monosulfate and 4-andorsten- $3 \beta, 17 \beta$-diol monosulfate were observed. Fig. 3(b) shows primary bile acid metabolism, whereby glycochenodeoxycholate and taurocholate were significantly decreased. Fig. 3(c) displays inositol metabolism whereby chiroinositol had a 26-fold increase after RB consumption. Fig. 3(d) showed significant increases in $\gamma$-carboxyethyl-hydroxychroman and pyridoxine (vitamin $\mathrm{B}_{6}$ ), which are metabolites in the tocopherol and vitamin $\mathrm{B}_{6}$ metabolic pathways, respectively.

Fig. 4(a) showed significantly perturbed AGE metabolism with N6-caboxymethyllysine decreasing 2-fold compared with baseline for the RB group. Fig. 4(b) displays the nine metabolites from the leucine, isoleucine and valine metabolic pathway with significant change in RB consumers compared with baseline ( $\beta$-hydroxyisovaleroylcarnitine, $\beta$-hydroxyisovalerate, 4-methyl-2-oxopentanoate, 3-methyl-2-oxobutyrate, 3-methyl2-oxovalerate, allo-isoleucine, ethylmalonate, isovalerylglycine and $N$-acetylleucine). Using this pathway visualisation tool, $\mathrm{N}$-acetylvaline (0.64-fold) and $\mathrm{N}$-acetylisoleucine (0.54-fold) had decreased expression and tiglyl-carnitine (C5:1-DC) (41-fold) increased at 4 weeks, and was trending towards significance compared with baseline in RB consumers. Fig. 4(c) shows the methionine, cysteine, $S$-adenosyl methionine and taurine metabolic pathways with 4-methylthio-2-oxobutanoate, $\mathrm{N}$ acetylmethionine sulfoxide and $\mathrm{N}$-acetylmethionine significantly decreased and cystathionine and $N$-formylmethionine increased. Fig. 4(d) displays the urea cycle; arginine and proline metabolism pathways and significantly decreased homocitrulline, $N$-methylproline and citrulline metabolites.

\section{Metabolite profile and pathway distinctions between control and rice bran consumers}

Compared with control diet, thirty-nine stool metabolites (sixteen of which had higher expression in RB consumers) were significantly different in the RB group after 4 weeks of consumption. This includes 28 -fold and 14.5-fold increased expression of the phytochemicals hesperidin and narirutin, respectively, in the stool of $\mathrm{RB}$ consumers compared with control diet. Table 2 features nineteen stool metabolites that were also increased in the RB 4 weeks/RB baseline statistical comparison (Table 1). These stool metabolites included lower expressed amino acid metabolites $S$-methylglutathione (3-fold), formimoglutamate (5-fold), 4-methyl-2-oxopentanoate (3-fold), 3-methyl-2-oxobutyrate (3-fold), 3-methyl-2-oxovalerate (3-fold), allo-isoleucine (3-fold), $\mathrm{N}$-acetylleucine (2-fold), 4-methylthio2-oxobutanoate (5-fold), 4-hydroxyphenylpyruvate (3-fold) and phenylpyruvate (3-fold), and the phytochemicals hesperidin (37-fold), narirutin (15-fold), diosmetin (5-fold), eriocitrin (2-fold) and solanidine (33-fold) than was observed compared with control diet consumers after 4 weeks of RB dietary intervention and over time. In addition, we observed higher amino acid metabolites expressed compared with control diet after 4 weeks and over time in RB consumers, such as xanthurenate (4-fold), the vitamin metabolite pyridoxine (vitamin $\mathrm{B}_{6}$; 3-fold), 2-hydroxyhippurate (salicylurate; 9-fold) and diosmin (7-fold).

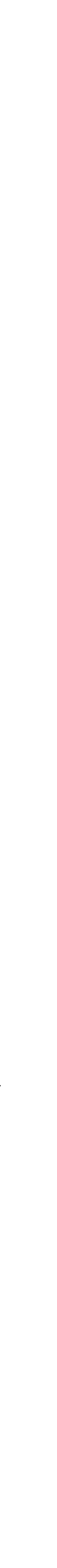




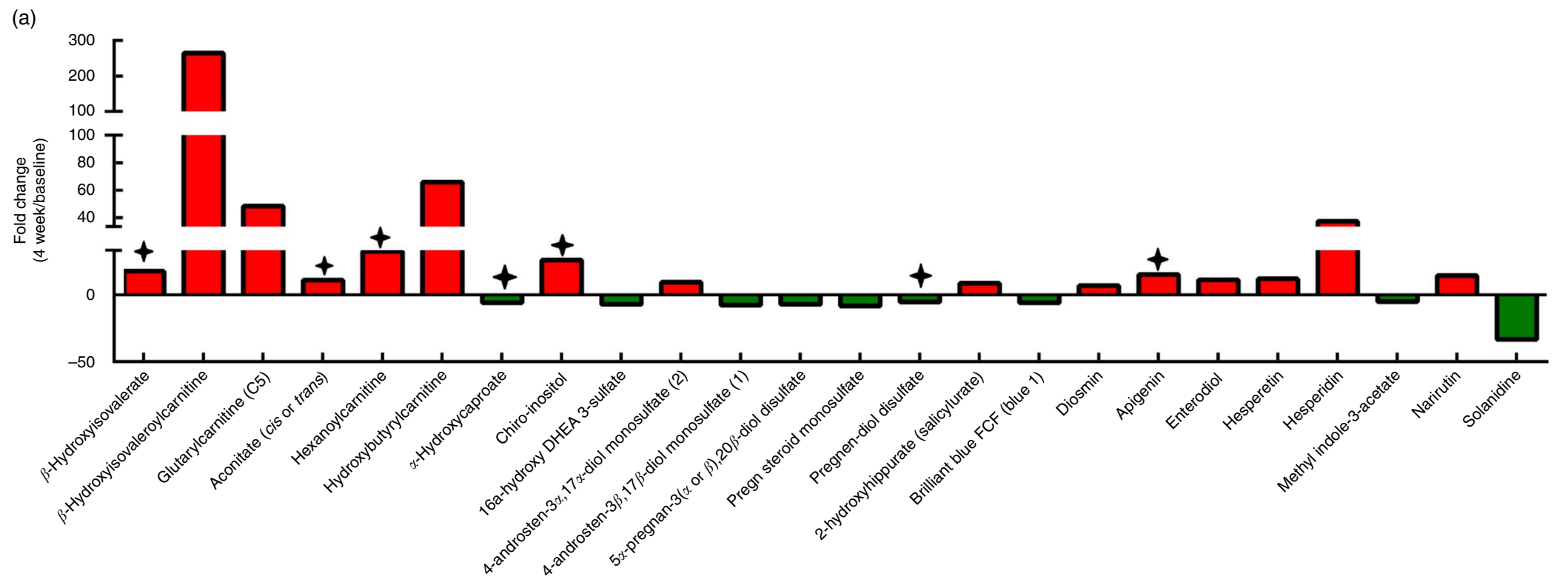

(b)

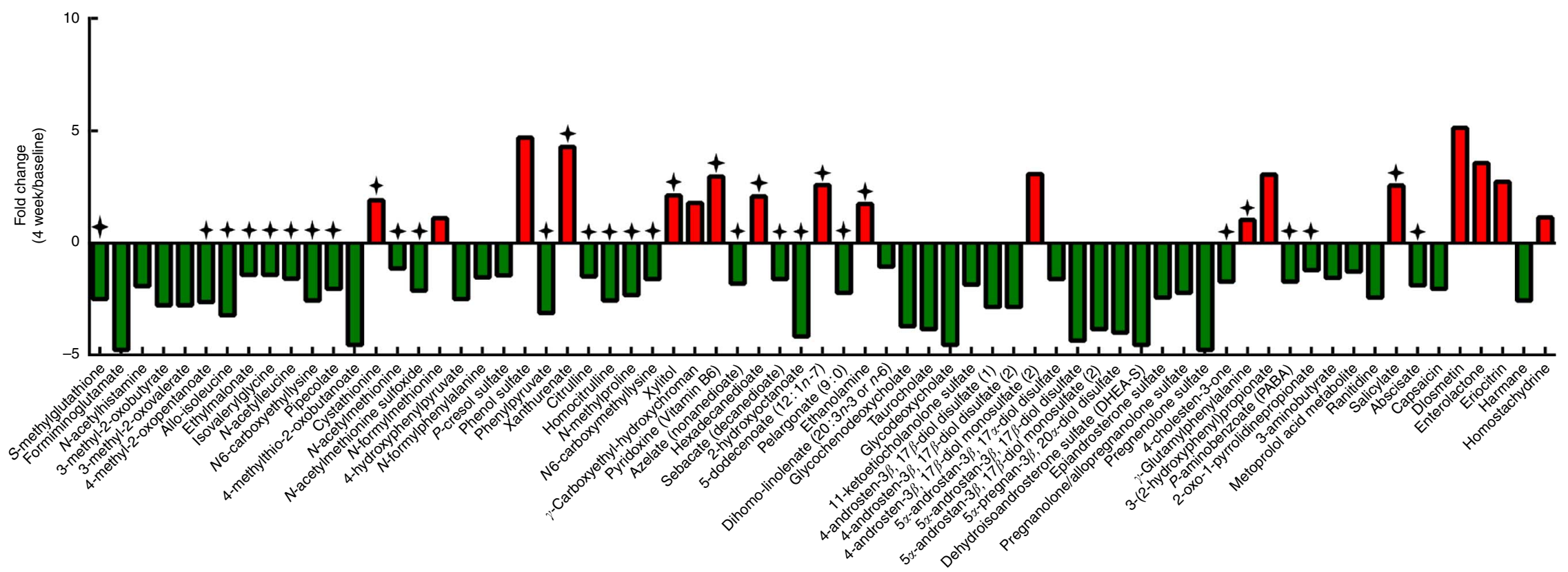

Fig. 1. Stool metabolite profile differences in the rice bran group over time. (a) Stool metabolites that were significantly different between 4 -week and baseline time points with fold change values $\geq 5$.00 and (b) stool metabolites that were significantly different between 4 -week and baseline time points and had fold change values $<5 \cdot 00$. + , Metabolites with significance and were identified in the rice bran food metabolome (see online Supplementary Table S3). 
(a)

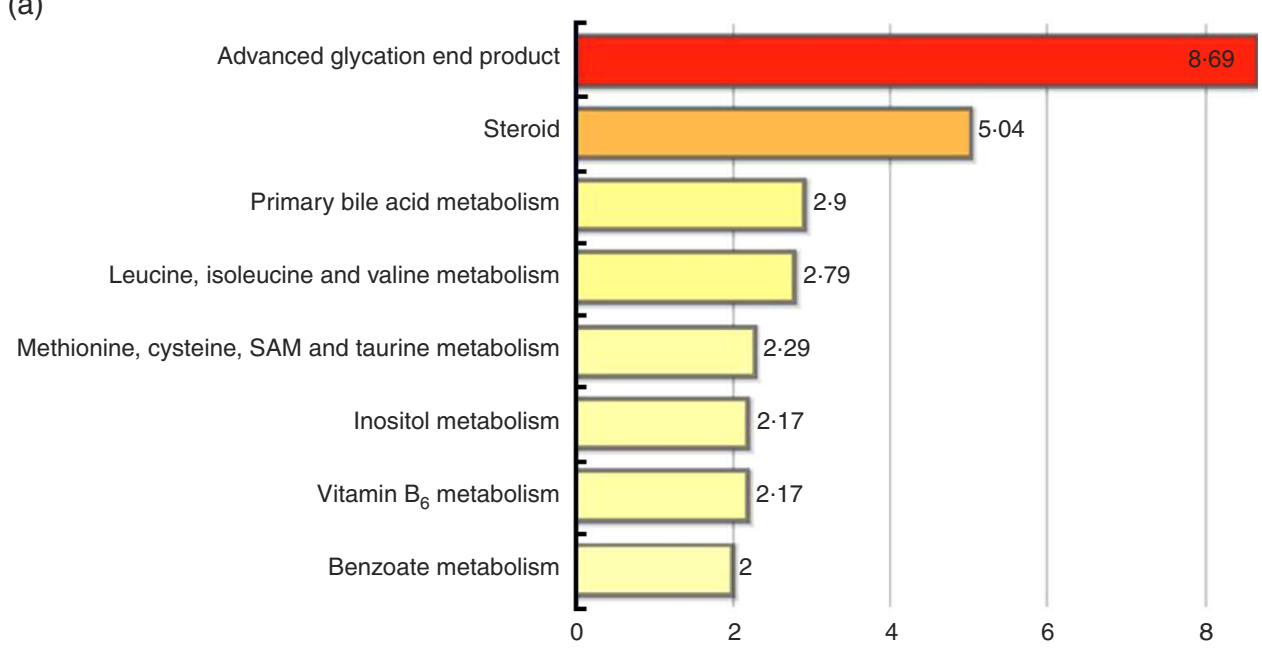

(b)

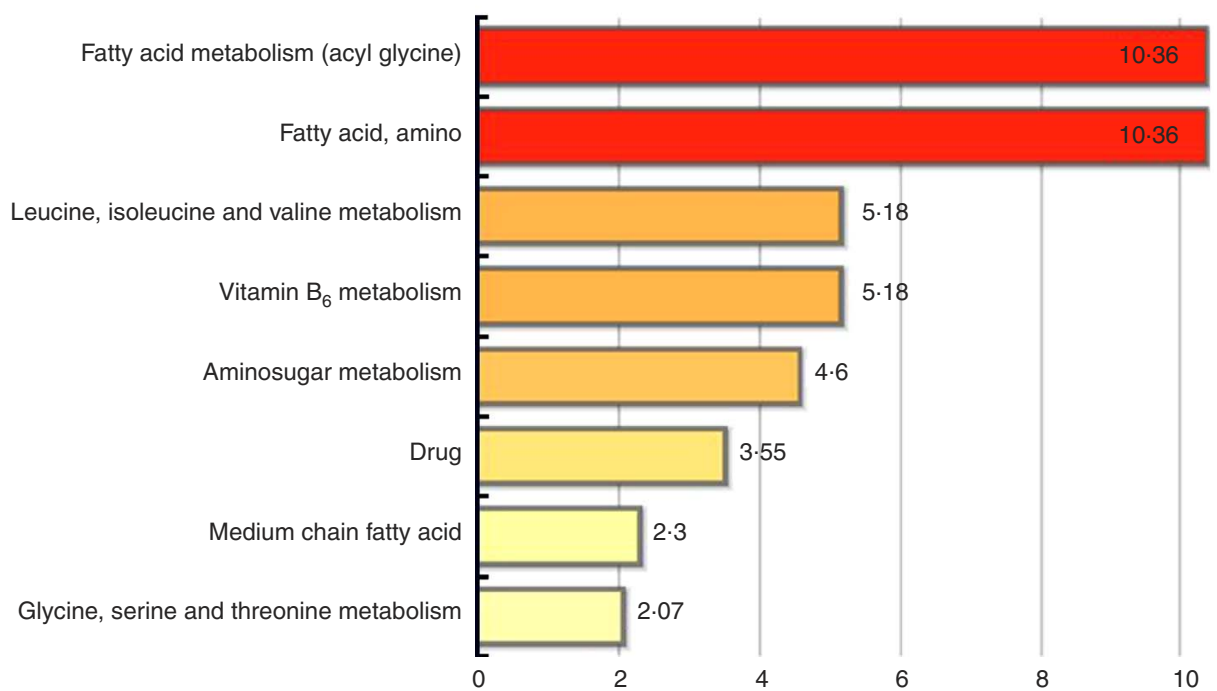

Fig. 2. Pathway set enrichment scores for stool metabolome. (a) Metabolic pathways in rice bran over time that were significantly different between 4-week and baseline time points and (b) rice bran diet compared with control diet at 4 weeks. Pathway set enrichment scores were determined as defined in the 'Methods' section, and are presented for pathways with scores $\geq 2$. SAM, S-adenosyl methionine.

There was modulation of eight metabolic pathways between RB and control diet consumers at 4 weeks. Fig. 2(b) shows fatty acid metabolism (pathway impact score of 10.36), leucine, isoleucine and valine metabolism (5.18), vitamin $\mathrm{B}_{6}$ metabolism (5.18), aminosugar metabolism (4.6), drug metabolism (3.55), medium-chain fatty acid (2.3) and glycine, serine and threonine metabolism (2.07).

There were forty-one metabolites with significant stool metabolome differences between CRC survivors consuming a control diet at 4 weeks compared with baseline. These control diet modifications in stool are listed in the online Supplementary Table S2. Increased 3-(2-hyroxyphenyl)propionate, salicylate, apigenin and enterolactone and decreased abscisate were observed among both diet groups at 4 weeks. N6carboxymethyllysine showed increased relative abundance after 4 weeks of consumption in control diet consumers, and a decreased relative abundance in RB consumers (Table 1 and online Supplementary Table S2).

\section{Rice bran food metabolome}

The RB food metabolome demonstrates extensive phytochemical diversity and provides an opportunity to look for cross-over with the stool metabolome, following consumption. The RB food metabolome was analysed for comparison with stool metabolite profiles. There were 453 metabolites identified from RB that were classified into seventy-four metabolic pathways. The online Supplementary Table S3 lists the RB metabolites and the metabolic pathway classifications identified in the stool of the RB group. Additional details on the RB metabolome can be found in Zarei et al. ${ }^{(28)}$.

\section{Overlap between the stool and rice bran food metabolomes}

The thirty-nine significantly changed stool metabolites from RB consumers that were also present in the RB food metabolome are shown in Fig. 1(a) and (b). These included 
(a)

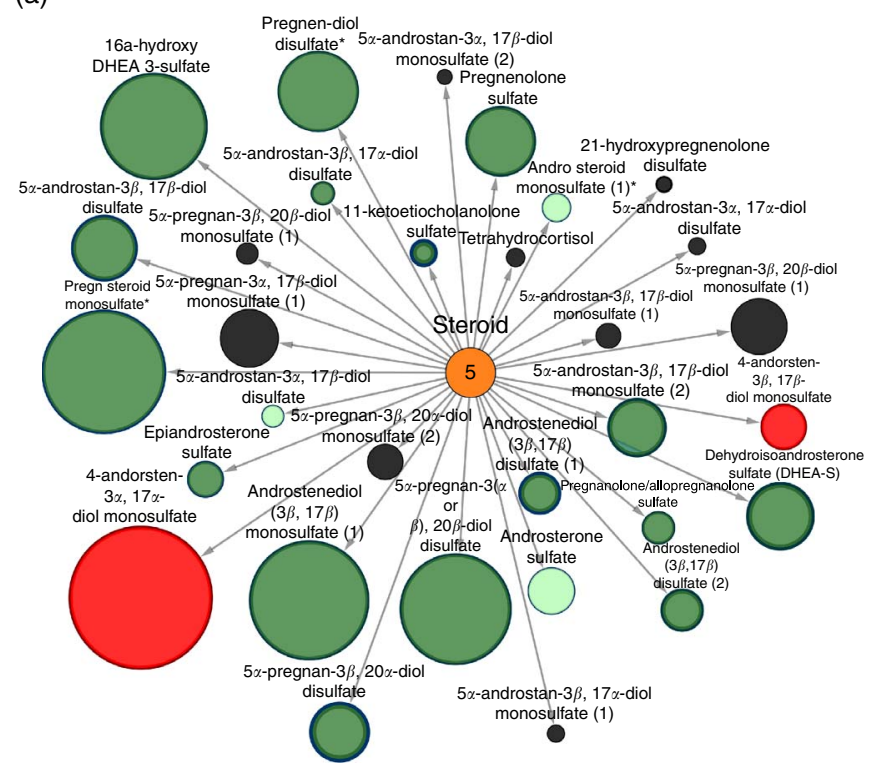

(c)

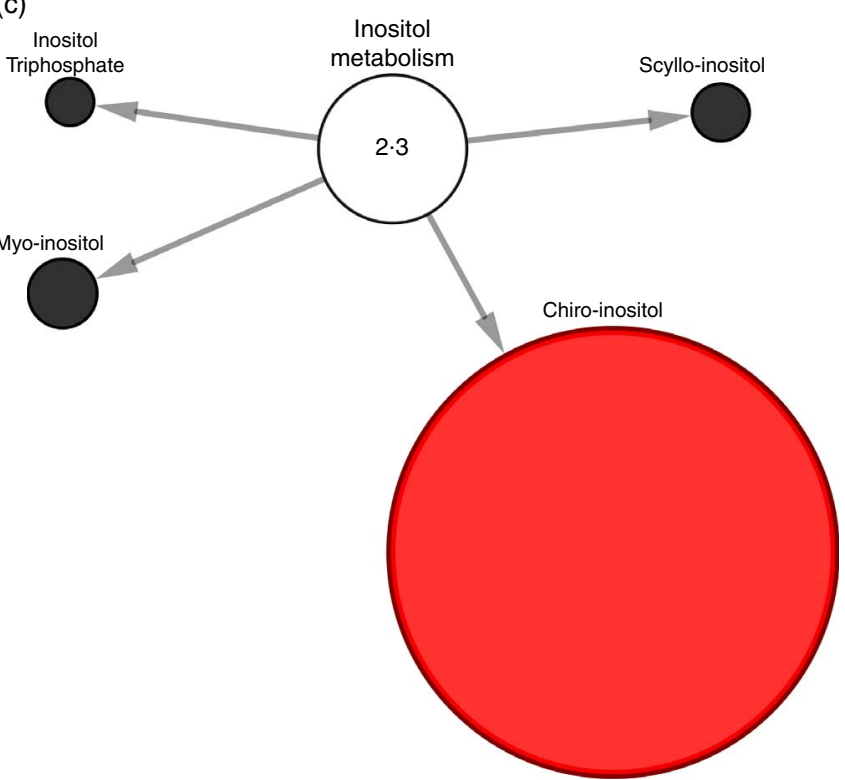

(b)

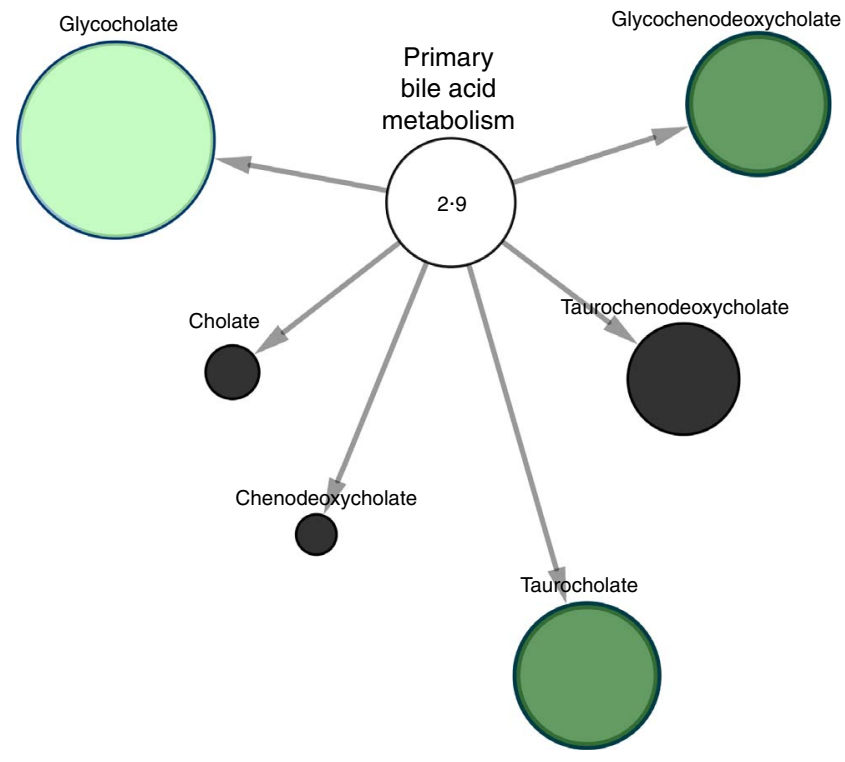

(d)

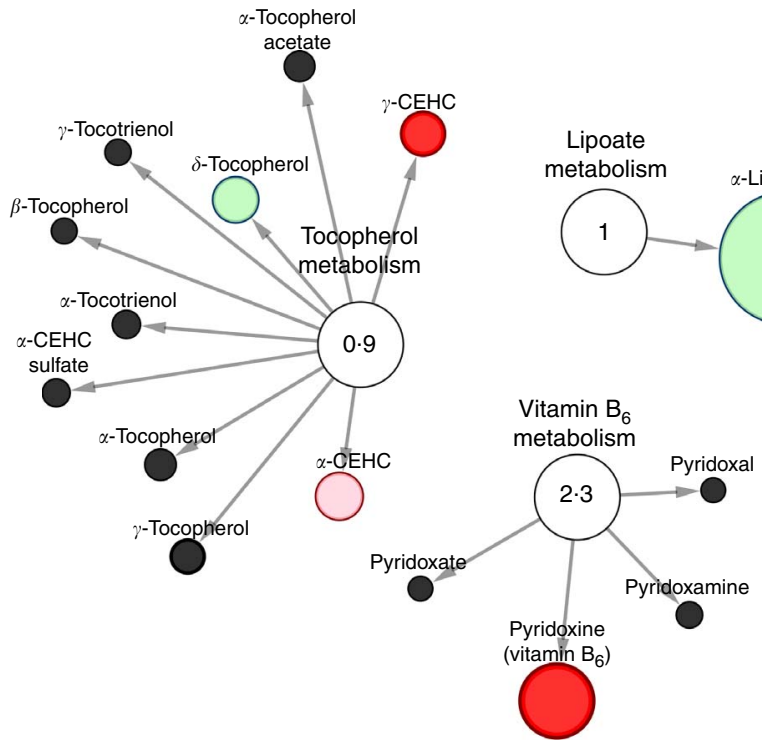

Fig. 3. Stool metabolomic pathway network analysis of lipid, cofactor and vitamin metabolites significantly different in rice bran consumers at 4 weeks compared with baseline. Pathway specific network views (Cytoscape) are presented for (a) steroid metabolism, (b) primary bile acid metabolism, (c) inositol metabolism, (d) cofactors and vitamin metabolism. Each metabolite is represented as a node, extending from a central metabolic pathway (with pathway impact score). A pathway set enrichment score $\geq 2$ implies that pathway contains more metabolites of statistical significance relative to the entire study. The size of a node is proportional to the fold change between baseline and 4-week time points. A node coloured red represents metabolites with significantly $(P \leq 0.05)$ higher expression at 4 weeks compared with baseline. A node coloured dark green represents metabolites with significantly lower expression at 4 weeks compared with baseline. Nodes coloured pink or light green represent metabolites trending towards significance $(0.05<P \leq 0.1)$ with higher expression at 4 weeks and lower expression at 4 weeks, respectively. CEHC, carboxyethyl-hydroxychroman.

N6-carboxymethyllysine, an AGE, which decreased by 2-fold in RB consumers' stool over 4 weeks. Apigenin (15-fold increased) and abscisate (2-fold decreased) were also present in the RB food metabolome, as well as the inositol metabolite chiroinositol, which increased 26-fold over time in the RB stool metabolome. Xanthurenate, a tryptophan metabolite, increased 4-fold in stool after RB consumption and was present in the RB. Pyridoxine was another metabolite involved in vitamin $\mathrm{B}_{6}$ metabolism that increased in stool and was present in RB. Fatty acids azelate (nonanedioate) (2-fold decreased), hexadecanedioate (2-fold increased), sebacate (decanedioate) (2-fold decreased), 2-hydroxyoctanoate (4-fold decreased) and $\alpha$-hydroxycaproate (6-fold decreased) also had dual significance for presence in the RB food metabolome (Fig. 1). Furthermore, 2-hydroxyhippurate (salicylurate) was expressed in the RB food metabolome, as well as after 4 weeks of $\mathrm{RB}$ consumption and when compared with the control diet. 
(a)

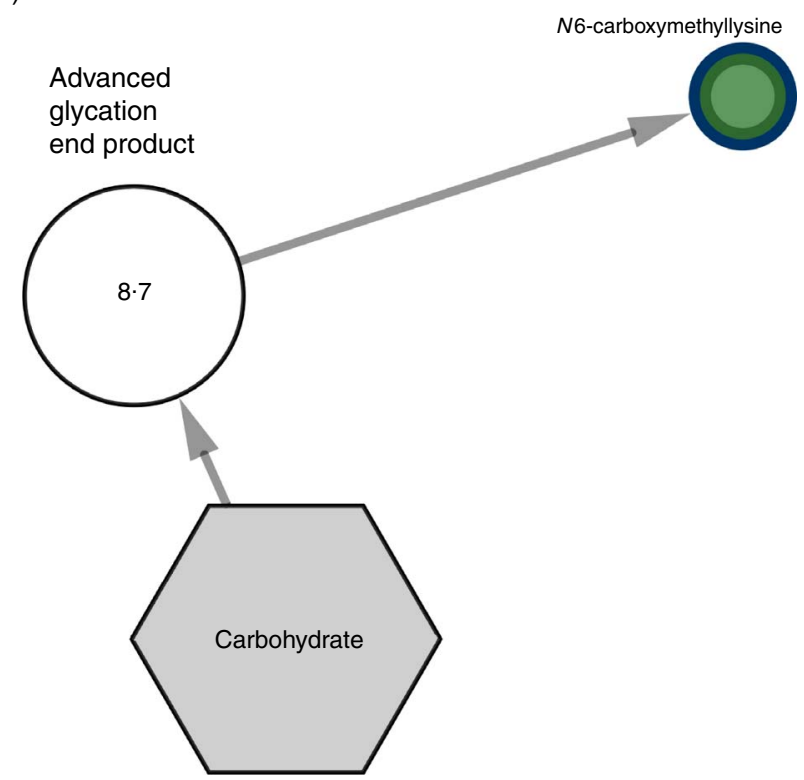

(c)

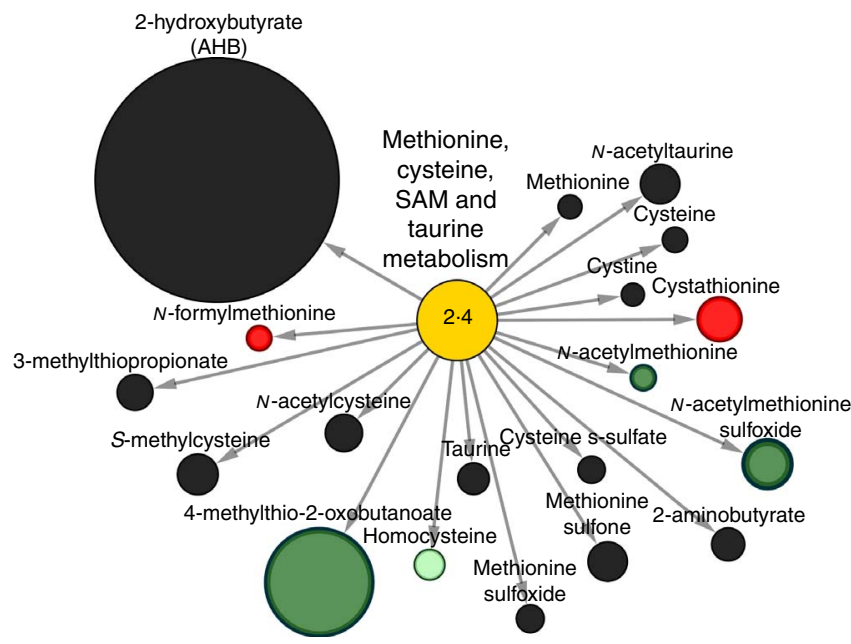

(b)

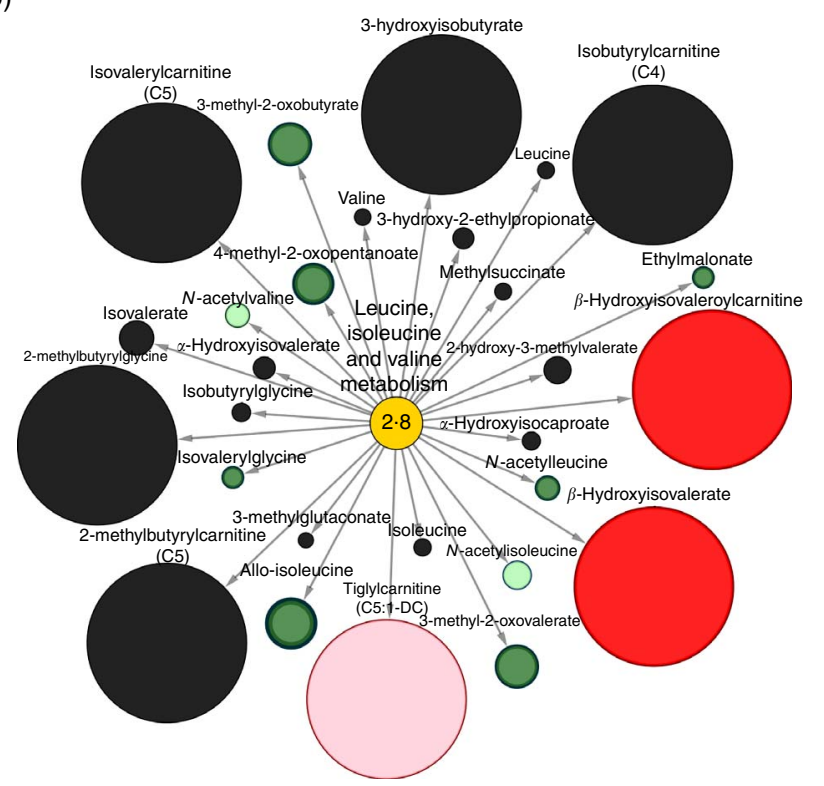

(d)

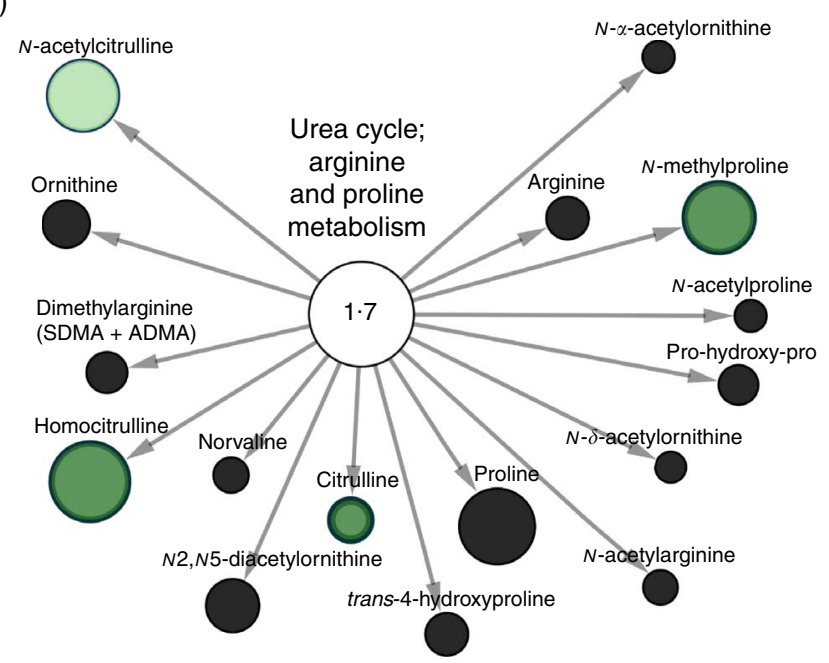

Fig. 4. Stool metabolomic pathway network analysis of carbohydrate and amino acid metabolites significantly different in rice bran consumers at 4 weeks compared with baseline. Pathway specific network views (Cytoscape) are presented for (a) advanced glycation end product metabolism, (b) leucine, isoleucine and valine metabolism, (c) methionine, cysteine, S-adenosyl methionine (SAM) and taurine metabolism, (d) urea cycle; arginine and proline metabolism. Each metabolite is represented as a node, extending from a central metabolic pathway (with pathway impact score). A pathway set enrichment score $\geq 2$ implies that pathway contains more metabolites of statistical significance relative to the entire study. The size of a node is proportional to the fold change between baseline and 4-week time points. A node coloured red represents metabolites with significantly $(P \leq 0.05)$ higher expression at 4 weeks compared with baseline. A node coloured dark green represents metabolites with significantly lower expression at 4 weeks compared with baseline. Nodes coloured pink or light green represent metabolites trending towards significance $(0.05<P \leq 0.1)$ with higher expression at 4 weeks and lower expression at 4 weeks, respectively.

\section{Discussion}

The primary objective of this study was to understand the stool metabolite profile differences between CRC survivors consuming a diet supplemented with RB for 4 weeks compared with a control group. These findings revealed that the consumption of $\mathrm{RB}$ leads to significant up-regulation of molecules from the carbohydrate, lipid, amino acid and cofactors and vitamin metabolic pathways in CRC survivors (Fig. 2-4). The ninetythree stool metabolites and eight metabolic pathways analysed within the context of the RB food metabolome may have functional potential for dietary chemoprevention and inform nutritional intakes in CRC survivors. Furthermore, multiple stool metabolites that originate from consumption of RB phytochemicals were identified.

Two stool metabolites with the highest degree of fold change between baseline and 4-week time points in RB consumers were $\beta$-hydroxyisovaleroylcarnitine and $\beta$-hydroxyisovalerate (a 265-fold and 18-fold, increase after consumption, respectively). Currently, no data have been published on $\beta$-hydroxyisovaleroylcarnitine and cancer, including CRC and limited data exists for $\beta$-hydroxyisovalerate. $\beta$-Hydroxyisovalerate has been used as a biomarker for lung cancer in a urinary metabolomics study ${ }^{(29)}$. This compound is closely related to 
Table 2. Stool metabolites distinguishing rice bran $(n 9)$ and control diet $(n 10)$ groups at 4 weeks

\section{Carbohydrate}

Cofactors and

vitamins

Lipid

Other

\begin{tabular}{|c|c|c|}
\hline Super pathways & Metabolic pathway & Metabolite \\
\hline \multirow[t]{13}{*}{ Amino acid } & $\begin{array}{l}\text { Glycine, serine and threonine } \\
\text { metabolism }\end{array}$ & Allo-threonine \\
\hline & Histidine metabolism & Formiminoglutamate \\
\hline & \multirow{6}{*}{$\begin{array}{l}\text { Leucine, isoleucine and valine } \\
\text { metabolism }\end{array}$} & Methylsuccinate \\
\hline & & $\begin{array}{l}\text { 4-methyl-2-oxopentanoate } \\
\text { 3-methyl-2-oxobutyrate }\end{array}$ \\
\hline & & 3-methyl-2-oxovalerate \\
\hline & & $\mathrm{N}$-acetylisoleucine \\
\hline & & $\mathrm{N}$-acetylleucine \\
\hline & & Allo-isoleucine \\
\hline & Lysine metabolism & 2-aminoadipate \\
\hline & $\begin{array}{l}\text { Methionine, cysteine, } S \text {-adenosyl } \\
\text { methionine and taurine metabolism }\end{array}$ & 4-methylthio-2-oxobutanoate \\
\hline & $\begin{array}{l}\text { Phenylalanine and tyrosine } \\
\text { metabolism }\end{array}$ & $\begin{array}{l}\text { 4-hydroxyphenylpyruvate } \\
\text { Phenylpyruvate }\end{array}$ \\
\hline & Tryptophan metabolism & Xanthurenate \\
\hline & $\begin{array}{l}\text { Urea cycle; arginine and proline } \\
\text { metabolism }\end{array}$ & $\mathrm{N}$-acetylcitrulline \\
\hline Carbohydrate & Aminosugar metabolism & $\begin{array}{l}\mathrm{N} \text {-acetylglucosamine } \\
\mathrm{N} \text {-acetylmuramate }\end{array}$ \\
\hline $\begin{array}{l}\text { Cofactors and } \\
\text { vitamins }\end{array}$ & Vitamin $\mathrm{B}_{6}$ metabolism & Pyridoxine (vitamin $B_{6}$ ) \\
\hline \multirow[t]{7}{*}{ Lipid } & Fatty acid metabolism(acyl glycine) & Valerylglycine \\
\hline & & $\begin{array}{l}\text { Heptanoyl glycine } \\
\text { Hexanoylglycine }\end{array}$ \\
\hline & Fatty acid, amino & 2-aminooctanoate \\
\hline & Lysolipid & 1-stearoylglycerophosphocholine (18:0) \\
\hline & Medium-chain fatty acid & 10-undecenoate $(11: 1 n-1)$ \\
\hline & Monoacylglycerol & 2-stearoylglycerol (2-monostearin) \\
\hline & PUFA ( $n-3$ and $n-6)$ & Docosadienoate $(22: 2 n-6)$ \\
\hline Nucleotide & $\begin{array}{l}\text { Pyrimidine metabolism, uracil } \\
\text { containing }\end{array}$ & 5-hydroxymethyluracil \\
\hline \multirow[t]{11}{*}{ Other } & \multirow[t]{7}{*}{ Xenobiotics } & 2-hydroxyhippurate (salicylurate) \\
\hline & & Metoprolol \\
\hline & & Diosmin \\
\hline & & Famotidine \\
\hline & & Diltiazem \\
\hline & & Methotrexate \\
\hline & & Fluoxetine \\
\hline & \multirow[t]{4}{*}{ Phytochemical } & Hesperidin \\
\hline & & Narirutin \\
\hline & & Diosmetin \\
\hline & & Eriocitrin \\
\hline
\end{tabular}

Control
relative
abundance

\begin{tabular}{|c|c|c|c|}
\hline 3.3772 & 0.5938 & 0.0201 & $0.18 \downarrow$ \\
\hline $2 \cdot 6375$ & 0.5645 & 0.0159 & $0.11 \downarrow$ \\
\hline 1.6559 & 1.4789 & 0.0429 & $1.87 \uparrow$ \\
\hline 1.7672 & 0.6699 & 0.004 & $0.26 \downarrow$ \\
\hline 1.8088 & 0.654 & 0.0083 & $0.23 \downarrow$ \\
\hline 1.7205 & 0.6219 & 0.0044 & $0.2 \downarrow$ \\
\hline 1.7169 & 0.9344 & 0.0388 & $0.18 \downarrow$ \\
\hline 1.265 & 0.7912 & 0.0376 & $0.15 \downarrow$ \\
\hline 2.5098 & 0.7683 & 0.0181 & $0.11 \downarrow$ \\
\hline 1.5763 & 1.1657 & 0.0192 & $1.68 \uparrow$ \\
\hline 0.8167 & 0.1811 & 0.0073 & $0.06 \downarrow$ \\
\hline 1.3693 & 0.5473 & 0.0032 & $0.31 \downarrow$ \\
\hline 1.643 & 0.5299 & 0.0017 & $0.19 \downarrow$ \\
\hline 0.3761 & 1.607 & $2 \cdot 85 \mathrm{E}-05$ & $10.81 \uparrow$ \\
\hline 0.8907 & 0.3838 & 0.0016 & $0.25 \downarrow$ \\
\hline 0.5458 & 0.4927 & 0.0422 & $0.2 \downarrow$ \\
\hline 0.8465 & 1.0116 & 0.0497 & $0.3 \downarrow$ \\
\hline $2 \cdot 3113$ & 6.8453 & 0.0034 & $6.74 \uparrow$ \\
\hline $1 \cdot 1844$ & 0.8156 & 0.0285 & $0.12 \downarrow$ \\
\hline 0.0201 & 0.0201 & 0.0198 & $0.02 \downarrow$ \\
\hline 0.8351 & 0.5023 & 0.0461 & $0.02 \downarrow$ \\
\hline 0.5119 & 0.4718 & 0.0309 & $0.17 \downarrow$ \\
\hline 0.413 & 0.7588 & 0.0307 & $0.07 \downarrow$ \\
\hline 0.8558 & 0.5173 & 0.0091 & $0.3 \downarrow$ \\
\hline 0.9148 & 1.1927 & 0.036 & $0.5 \downarrow$ \\
\hline 0.6816 & 1.2882 & 0.0149 & $0.24 \downarrow$ \\
\hline 0.1535 & 0.3954 & 0.0488 & $2.58 \uparrow$ \\
\hline 0.4121 & 3.6062 & 1.47E-05 & $3.75 \uparrow$ \\
\hline 0.46 & 0.4479 & 0.0361 & $73.43 \uparrow$ \\
\hline 0.0974 & 0.6707 & 0.0021 & $4.68 \uparrow$ \\
\hline 0.0866 & 0.3476 & 0.0488 & $4.01 \uparrow$ \\
\hline 0.3985 & 0.5704 & 0.0488 & $1.43 \uparrow$ \\
\hline 0.7719 & 0.8371 & 0.0488 & $1.08 \uparrow$ \\
\hline 0.4939 & 0.6136 & 0.0488 & $1.06 \uparrow$ \\
\hline 0.3687 & 13.7199 & 0.0026 & $28.44 \uparrow$ \\
\hline 0.0307 & 0.4461 & 0.0032 & $14.53 \uparrow$ \\
\hline 0.2638 & 1.3527 & 0.035 & $3.97 \uparrow$ \\
\hline 0.2541 & 0.6921 & 0.0044 & $2.72 \uparrow$ \\
\hline 2.5996 & 0.0778 & 0.016 & $0.03 \downarrow$ \\
\hline
\end{tabular}

$\uparrow$, Metabolites with significantly $(P \leq 0.05)$ higher expression in rice bran consumers at 4 weeks when compared with control at 4 weeks (metabolite ratio of $\geq 1.00)$; $\downarrow$, metabolites with significantly $(P \leq 0.05)$ lower expression in rice bran consumers at 4 weeks when compared with control at 4 weeks (metabolite ratio of $<1.00)$.

biotin-mediated metabolism and has been detected in plasma metabolome of CRC patients ${ }^{(30,31)}$. Dual positive detection herein from the stool and RB food metabolome (online Supplementary Table S3) merits continued investigation. The large differences further suggest the need to evaluate the functional potential of RB modifications to leucine, isoleucine and valine in metabolism for enhanced colonic health.

Pyridoxine (vitamin $\mathrm{B}_{6}$ ) increased 3-fold in the stool at 4 weeks in RB consumers compared with baseline (Table 1), as well as compared with control at end of study (Table 2) and was present in the RB food metabolome (online Supplementary Table S3). Dietary nutritional intake analysis via weekly food logs also showed an increase in vitamin $\mathrm{B}_{6}$ before analysing the stool metabolome ${ }^{(21)}$. Vitamin $\mathrm{B}_{6}$ is a water-soluble vitamin present in the human body as pyridoxine, pyridoxal and pyridoxamine and serves as a coenzyme in folate metabolism, which has associations with CRC carcinogenesis ${ }^{(32)}$. Vitamin $\mathrm{B}_{6}$ has potential to influence $\mathrm{CRC}$ risk in various ways, including DNA synthesis and methylation and reduction of inflammation, cell proliferation and oxidative stress ${ }^{(33)}$. Recommended intake is $1.7 \mathrm{mg} / \mathrm{d}$ for men and $1.5 \mathrm{mg} / \mathrm{d}$ for women, with $30 \mathrm{~g}$ of $\mathrm{RB}$ providing $1.2 \mathrm{mg} / \mathrm{d}^{(21)}$. Furthermore, dose-dependent associations were found between CRC risk and dietary vitamin $\mathrm{B}_{6}$ intake ${ }^{(34)}$. Moreover, xanthurenate, a tryptophan metabolite tied to vitamin $\mathrm{B}_{6}$ maintenance ${ }^{(35)}$, was significantly increased over time in RB consumers and when compared with control diet group. Based on the results and what is known about pyridoxine in $\mathrm{CRC}$ literature, we postulate that vitamin $\mathrm{B}_{6}$ 
represents a diet modifiable biomarker of $\mathrm{RB}$ intake in CRC survivors and should be confirmed with a targeted investigation in a larger cohort.

Phytochemicals related to citrus intake include hersperidin, eriocitrin and narirutin were significantly increased over time with RB consumption. Hesperidin has shown to alleviate oxidative stress and down-regulate proliferative inflammatory markers in azoxymethane-induced colon carcinogenesis in mice $^{(36)}$. Eriocitrin has shown significance in multiple analyses and has anti-oxidative properties in vitro ${ }^{(37)}$. Narirutin, has evidence for anti-oxidative ${ }^{(38)}$, anti-inflammatory ${ }^{(39)}$ and antiallergic activities ${ }^{(40)}$. These three compounds were increased with RB diet consumption in our study and may be related to RB metabolism changes by the host. Solanidine, a phytochemical of importance to CRC survivors, is a steroidal alkaloid that has anticancer activities against human colon HCT- 8 and HT-29 cancer cells ${ }^{(41)}$. The phytochemical diosmetin and a closely related microbiota metabolism-driven derivative, diosmin, have shown oxidative activity against cancer cells (Colon205), inducing apoptosis in Colo320 CRC cells, as well as reducing the multiplicity of $\mathrm{CRC}$ neoplasms in azoxymethanetreated mice ${ }^{(42,43)}$. Phytochemical modulation and host metabolites in stool following $\mathrm{RB}$ consumption represents a novel chemopreventive mechanism that should be further explored.

N6-carboxymethyllysine is an AGE which are a group of heterogeneous macromolecules that are formed by the nonenzymatic glycation of proteins, lipids and nucleic acids ${ }^{(44)}$. There are two main sources of AGE in humans: food and endogenously produced exposures ${ }^{(45)}$. AGE are typically generated in higher rates in diabetics due to altered glucose metabolism $^{(46)}$. In our study, N6-carboxymethlysine was decreased with $\mathrm{RB}$ consumption over time and increased in control diet consumers at 4 weeks, which is consistent with rice bran and whole grain brown rice having the ability to lower glucose and serum lipids in Type I and II diabetics ${ }^{(47-49)}$. Another stool metabolite of importance to diabetes control and CRC prevention is 2-hydroxyhippurate (salicylurate). This compound increased by 9 -fold following RB consumption after 4 weeks, and had an increased fold change of 3.75 compared with control at 4 weeks (Tables 1 and 2). 2-hydroxyhippurate (salicylurate) is a metabolite of aspirin, and has not only shown the ability to prevent CVD complications in diabetes mellitus patients, but also has demonstrated high efficacy in reducing incidence of colorectal neoplasias ${ }^{(50-52)}$. Moreover, faecal 2hydroxyhippurate (salicylurate) levels were associated with a decreased risk of $\mathrm{CRC}$ in a prior faecal metabolomics investigation $^{(53)}$. Therefore, we postulate that decreased AGE and increased 2-hydroxyhippurate (salicylurate) levels in stool due to RB consumption represent potential mechanisms for chemoprevention. This dietary compound has relevance as candidate markers of glucose, lipid and xenobiotic metabolism changes.

The individual metabolites, as well as the network of metabolic pathways that were modulated by RB consumption, identified primary bile acid metabolism with a pathway impact score of 2.9 . Primary bile acids included glycochenodeoxycholate and taurocholate that were decreased nearly 4-fold in stool after 4 weeks of RB consumption. In addition, there was a 5-fold decreased expression of glycodeoxycholate, a secondary bile acid. Primary bile acids are synthesised by the human liver and undergo transformation to secondary bile acids by the microbiota ${ }^{(54)}$. Secondary bile acids have associations with reactive $\mathrm{O}_{2} / \mathrm{N}$ species generation and oxidative DNA damage $^{(55)}$. The reduction of bile acid metabolites (primary and secondary) in the stool metabolome of CRC survivors is consistent with healthy adults ${ }^{(15)}$ and was identified as a novel mechanism for RB chemoprevention that should be further explored.

Nutrition-based therapies have proven effective for improving long-term prognosis in CRC patients ${ }^{(56,57)}$. Increasing dietary fibre has been inversely correlated with CRC risk ${ }^{(58)}$, and $\mathrm{RB}$ represents a rich source of dietary fibre that can be successfully incorporated into the diet ${ }^{(15,21,22)}$. This unique metabolome analytical approach was to dually investigate the stool metabolite profiles and RB food metabolome, where the online Supplementary Table S3 illustrates the thirty-nine metabolites identified in both the $\mathrm{RB}$ metabolome and stool metabolome of $\mathrm{RB}$ consumers. $\beta$-Hydroxyisovalerate and pyridoxine represent two metabolites of potential dietary biomarkers of RB intake and identified in stool. Foodomics is a branch of metabolomics aimed at quantifying metabolite intakes and to correlate these exposures with biomarkers and health benefits ${ }^{(59)}$. The combined foodomics and stool metabolomics analytical approach with CRC survivors led to the identification metabolic pathway networks associated with dietary chemoprevention. Major strengths of this study were the confident metabolite identifications from a non-targeted stool and RB food metabolomics analysis. Study limitations included low sample size and high inter-group variation across individuals. Based on these results, we postulate that urine and blood matrices merit a similar analytical integration to determine specific, sensitive biomarkers of food intake and biological accessibility. The compelling findings from this subset of individuals included in this investigation provide rationale for $\mathrm{RB}$ consumption in larger studies and over longer periods of time to further analyse dietary biomarkers of intake, as well as to identify biomarkers of $\mathrm{CRC}$ recurrence and prevention, particularly in the colon tissue. Our findings demonstrate that the integration of food and stool metabolomes is useful for understanding dietary chemopreventive mechanisms for CRC prevention and control.

\section{Acknowledgements}

The authors would like to acknowledge the University of Colorado Health - North Oncology Research group, specifically Joann Lovins, Erica Dickson, Joanne O'Malia and Marlon Bazan, for helping with regulatory approvals, assistance with study recruitment, implementation, consents and coordination of sample collection. The authors thank Dr Anna McClung from the US Department of Agriculture for supplying the study intervention heat-stabilised rice bran; Dr Tiffany Weir for assistance in study design, food preparation and stool collection; and Iman Zarei for helping with proofreading. Katie Schmitz, Genevieve Forster, Allie Reava, Kerry Gundlach and 
Brianna Nervig provided technical support with study food preparations and nutrition analysis. Finally, the authors wish to thank all the study participants for their contribution to the trial.

This work was supported by the National Institutes Health National Cancer Institute (1R21CA161472), National Institute of Food and Agriculture (2016-67001-24538) and the University of Colorado Cancer Center - Division of Cancer Prevention and Control pilot award programme.

The authors' responsibilities were as follows - E. P. R. and R. J. B.: designed research and maintained study oversight; E. C. B.: conducted research; D. G. B.: analysed stool and rice bran for metabolites; D. G. B., E. C. B. and E. P. R. wrote paper; E. P. R. had primary responsibility for the final product. All authors read and approved the final manuscript.

None of the authors has any conflicts of interest to declare.

\section{Supplementary material}

For supplementary material/s referred to in this article, please visit https://doi.org/10.1017/S0007114517001106

\section{References}

1. Perera PST, Thompson RL \& Wiseman MJ (2012) Recent evidence for colorectal cancer prevention through healthy food, nutrition, and physical activity: implications for recommendations. Curr Nutr Rep 1, 44-54.

2. Li YH, Niu YB, Sun Y, et al. (2015) Role of phytochemicals in colorectal cancer prevention. World J Gastroenterol 21, 9262-9272.

3. Liu RH (2004) Potential synergy of phytochemicals in cancer prevention: mechanism of action. J Nutr 134, 3479S-3485S.

4. Ferlay J, Soerjomataram I, Ervik M, et al. (2014) GLOBOCAN 2012 v1.1, Cancer Incidence and Mortality Worldwide, IARC CancerBase no. 11. Lyon: International Agency for Research on Cancer. http://globocan.iarc.fr (accessed February 2017).

5. Slavin J (2004) Whole grains and human health. Nutr Res Rev 17, 99-110.

6. Windey K, De Preter V, Huys G, et al. (2015) Wheat bran extract alters colonic fermentation and microbial composition, but does not affect faecal water toxicity: a randomised controlled trial in healthy subjects. Br J Nutr 113, 225-238.

7. Katyama M, Yoshimi N, Yamada Y, et al. (2002) Preventive effect of fermented brown rice and rice bran against colon carcinogenesis in male F344 rats. Oncol Rep 9, 817-822.

8. Norazalina S, Norhaizan ME, Hairuszah I, et al. (2010) Anticarcinogenic efficacy of phytic acid extracted from rice bran on azoxymethane-induced colon carcinogenesis in rats. Exp Toxicol Pathol 62, 259-268.

9. Verschoyle RD, Greaves P, Cai H, et al. (2007) Evaluation of the cancer chemopreventive efficacy of rice bran in genetic mouse models of breast, prostate and intestinal carcinogenesis. Br J Cancer 96, 248-254.

10. Henderson AJ, Ollila CA, Kumar A, et al. (2012) Chemopreventive properties of dietary rice bran: current status and future prospects. Adv Nutr 3, 643-653.

11. Norris L, Malkar A, Horner-Glister E, et al. (2015) Search for novel circulating cancer chemopreventive biomarkers of dietary rice bran intervention in Apc(Min) mice model of colorectal carcinogenesis, using proteomic and metabolic profiling strategies. Mol Nutr Food Res 59, 1827-1836.
12. Phutthaphadoong S, Yamada Y, Hirata A, et al. (2010) Chemopreventive effect of fermented brown rice and rice bran (FBRA) on the inflammation-related colorectal carcinogenesis in ApcMin/ + mice. Oncol Rep 23, 53-59.

13. Islam MS, Nagasaka R, Ohara K, et al. (2011) Biological abilities of rice bran-derived antioxidant phytochemicals for medical therapy. Curr Top Med Chem 11, 1847-1853.

14. Sun W, Xu W, Liu H, et al. (2009) gamma-Tocotrienol induces mitochondria-mediated apoptosis in human gastric adenocarcinoma SGC-7901 cells. J Nutr Biochem 20, 276-284.

15. Sheflin AM, Borresen EC, Wdowik MJ, et al. (2015) Pilot dietary intervention with heat-stabilized rice bran modulates stool microbiota and metabolites in healthy adults. Nutrients 7, 1282-1300.

16. Martinez I, Lattimer JM, Hubach KL, et al. (2013) Gut microbiome composition is linked to whole grain-induced immunological improvements. ISME J 7, 269-280.

17. Komiyama Y, Andoh A, Fujiwara D, et al. (2011) New prebiotics from rice bran ameliorate inflammation in murine colitis models through the modulation of intestinal homeostasis and the mucosal immune system. Scand J Gastroenterol 46, 40-52.

18. Schmidt CG, Goncalves LM, Prietto L, et al. (2014) Antioxidant activity and enzyme inhibition of phenolic acids from fermented rice bran with fungus Rizhopus oryzae. Food Chem 146, 371-377.

19. Hullar MA, Burnett-Hartman AN \& Lampe JW (2014) Gut microbes, diet, and cancer. Cancer Treat Res 159, 377-399.

20. Ryan EP, Heuberger AL, Weir TL, et al. (2011) Rice bran fermented with Saccharomyces boulardii generates novel metabolite profiles with bioactivity. J Agric Food Chem 59, 1862-1870

21. Borresen EC, Brown DG, Harbison G, et al. (2016) A randomized controlled trial to increase navy bean or rice bran consumption in colorectal cancer survivors. Nutr Cancer $\mathbf{6 8}$, 1269-1280.

22. Sheflin AM, Borresen EC, Kirkwood JS, et al. (2016) Dietary supplementation with rice bran or navy bean alters gut bacterial metabolism in colorectal cancer survivors. Mol Nutr Food Res (Epublication ahead of print version 12 September 2016).

23. Heuberger AL, Lewis MR, Chen MH, et al. (2010) Metabolomic and functional genomic analyses reveal varietal differences in bioactive compounds of cooked rice. PloS ONE 5, e12915.

24. Brown DG, Rao S, Weir TL, et al. (2016) Metabolomics and metabolic pathway networks from human colorectal cancers, adjacent mucosa, and stool. Cancer Metab 4, 1-12.

25. Evans AM, DeHaven CD, Barrett T, et al. (2009) Integrated, nontargeted ultrahigh performance liquid chromatography/ electrospray ionization tandem mass spectrometry platform for the identification and relative quantification of the smallmolecule complement of biological systems. Anal Chem 81, 6656-6667.

26. Nyamundanda G, Gormley IC, Fan Y, et al. (2013) MetSizeR: selecting the optimal sample size for metabolomic studies using an analysis based approach. BMC Bioinformatics 14, 338 .

27. Blaise BJ (2013) Data-driven sample size determination for metabolic phenotyping studies. Anal Chem 85, 8943-8950.

28. Zarei I, Brown DG, Nealon NJ, et al. (2017) Rice bran metabolome contains amino acids, vitamins, cofactors, and phytochemicals with medicinal and nutritional properties. Rice. doi:10.1186/s12284-017-0157-2.

29. Silva C, Cavaco C, Perestrelo R, et al. (2014) Microextraction by packed sorbent (MEPS) and solid-phase microextraction (SPME) as sample preparation procedures for the metabolomic profiling of urine. Metabolites $\mathbf{4}, 71-97$. 
30. Fukui Y \& Itoh K (2010) A plasma metabolomic investigation of colorectal cancer patients by liquid chromatography-mass spectrometry. Open Anal Chem J 4, 1-9.

31. Mock NI, Malik MI, Stumbo PJ, et al. (1997) Increased urinary excretion of 3-hydroxyisovaleric acid and decreased urinary excretion of biotin are sensitive early indicators of decreased biotin status in experimental biotin deficiency. Am J Clin Nutr 65, 951-958.

32. Clayton PT (2006) $\mathrm{B}_{6}$-responsive disorders: a model of vitamin dependency. J Inherit Metab Dis 29, 317-326.

33. Zhang XH, Ma J, Smith-Warner SA, et al. (2013) Vitamin $\mathrm{B}_{6}$ and colorectal cancer: current evidence and future directions. World J Gastroenterol 19, 1005-1010.

34. Theodoratou E, Farrington SM, Tenesa A, et al. (2008) Dietary vitamin $\mathrm{B}_{6}$ intake and the risk of colorectal cancer. Cancer Epidemiol Biomarkers 17, 171-182.

35. Institute of Medicine (US) Standing Committee on the Scientific Evaluation of Dietary Reference Intakes and its Panel on Folate, Other B Vitamins, and Choline (1998) Dietary Reference Intakes for Thiamin, Riboflavin, Niacin, Vitamin B6, Folate, Vitamin B12, Pantothenic Acid, Biotin, and Choline. Washington, DC: National Academies Press.

36. Saiprasad G, Chitra P, Manikandan R, et al. (2013) Hesperidin alleviates oxidative stress and downregulates the expressions of proliferative and inflammatory markers in azoxymethaneinduced experimental colon carcinogenesis in mice. Inflamm Res 62, 425-440.

37. Miyake Y, Yamamoto K, Morimitsu Y, et al. (1997) Isolation of C-glucosylflavone from lemon peel and antioxidative activity of flavonoid compounds in lemon fruit. J Agric Food Chem $\mathbf{4 5}$, 4619-4623.

38. Tripoli E, La Guardia M, Giammanco S, et al. (2007) Citrus flavonoids: molecular structure, biological activity and nutritional properties: a review. Food Chem 104, 466-479.

39. Funaguchi N, Ohno Y, La BLB, et al. (2007) Narirutin inhibits airway inflammation in an allergic mouse model. Clin Exp Pharmacol Physiol 34, 766-770.

40. Park HY, Ha SK, Eom H, et al. (2013) Narirutin fraction from citrus peels attenuates alcoholic liver disease in mice. Food Chem Toxicol 55, 637-644.

41. Jiang QW, Chen MW, Cheng KJ, et al. (2016) Therapeutic potential of steroidal alkaloids in cancer and other diseases. Med Res Rev 36, 119-143.

42. Xie YY, Yuan D, Yang JY, et al. (2009) Cytotoxic activity of flavonoids from the flowers of Chrysanthemum morifolium on human colon cancer Colon205 cells. J Asian Nat Prod Res 11, 771-778.

43. Zheng Q, Hirose Y, Yoshimi N, et al. (2002) Further investigation of the modifying effect of various chemopreventive agents on apoptosis and cell proliferation in human colon cancer cells. J Cancer Res Clin Oncol 128, 539-546.

44. Fu MX, Requena JR, Jenkins AJ, et al. (1996) The advanced glycation end product, Nepsilon-(carboxymethyl)lysine, is a product of both lipid peroxidation and glycoxidation reactions. J Biol Chem 271, 9982-9986.
45. Uribarri J, Woodruff S, Goodman S, et al. (2010) Advanced glycation end products in foods and a practical guide to their reduction in the diet. J Am Diet Assoc 110, 911-916 e912.

46. Ahmed N (2005) Advanced glycation endproducts - role in pathology of diabetic complications. Diabetes Res Clin Pract 67, 3-21.

47. Kim TH, Kim EK, Lee MS, et al. (2011) Intake of brown rice lees reduces waist circumference and improves metabolic parameters in type 2 diabetes. Nutr Res 31, 131-138.

48. Qureshi AA, Sami SA \& Khan FA (2002) Effects of stabilized rice bran, its soluble and fiber fractions on blood glucose levels and serum lipid parameters in humans with diabetes mellitus Types I and II. J Nutr Biochem 13, 175-187.

49. C Chou TW, Ma CY, Cheng HH, et al. (2009) A rice bran oil diet improves lipid abnormalities and suppress hyperinsulinemic responses in rats with streptozotocin/ nicotinamide-induced type 2 diabetes. J Clin Biochem Nutr 45, 29-36.

50. Levy G (1980) Clinical pharmacokinetics of salicylates: a re-assessment. Br J Clin Pharmacol 10, Suppl. 2, 285S-290S.

51. Garcia-Albeniz X \& Chan AT (2011) Aspirin for the prevention of colorectal cancer. Best Pract Res Clin Gastroenterol 25, 461-472.

52. Pignone M, Alberts MJ, Colwell JA, et al. (2010) Aspirin for primary prevention of cardiovascular events in people with diabetes: a position statement of the American Diabetes Association, a scientific statement of the American Heart Association, and an expert consensus document of the American College of Cardiology Foundation. Circulation 121, 2694-2701

53. Goedert JJ, Sampson JN, Moore SC, et al. (2014) Fecal metabolomics: assay performance and association with colorectal cancer. Carcinogenesis 35, 2089-2096.

54. Sayin SI, Wahlstrom A, Felin J, et al. (2013) Gut microbiota regulates bile acid metabolism by reducing the levels of taurobeta-muricholic acid, a naturally occurring FXR antagonist. Cell Metab 17, 225-235.

55. Payne CM, Bernstein C, Dvorak K, et al. (2008) Hydrophobic bile acids, genomic instability, Darwinian selection, and colon carcinogenesis. Clin Exp Gastroenterol 1, 19-47.

56. Ravasco P, Monteiro-Grillo I \& Camila M (2012) Individualized nutrition intervention is of major benefit to colorectal cancer patients: long-term follow-up of a randomized controlled trial of nutritional therapy. Am J Clin Nutr 96, 1346-1353.

57. Ravasco P, Monteiro-Grillo I, Vidal PM, et al. (2005) Dietary counseling improves patient outcomes: a prospective, randomized, controlled trial in colorectal cancer patients undergoing radiotherapy. J Clin Oncol 23, 1431-1438.

58. Dahm CC, Keogh RH, Lentjes MAH, et al. (2010) Intake of dietary fats and colorectal cancer risk: prospective findings from the UK Dietary Cohort Consortium. Cancer Epidemiol 34, 562-567.

59. Ibanez C, Valdes A, Garcia-Canas V, et al. (2012) Global Foodomics strategy to investigate the health benefits of dietary constituents. J Chromatogr A 1248, 139-153. 